\title{
Involvement of the OsMKK4-OsMPK1 Cascade and its Downstream Transcrip- tion Factor OsWRKY53 in the Wounding Response in Rice
}

\author{
Seung Jin Yoo ${ }^{1}$, Su-Hyun Kim${ }^{1}$, Min-Jeong Kim ${ }^{1}$, Choong-Min Ryu ${ }^{2,3}$, Young Cheol Kim ${ }^{1}$, Baik Ho Cho ${ }^{1}$ and \\ Kwang-Yeol Yang ${ }^{1 *}$ \\ ${ }^{1}$ Department of Plant Biotechnology, College of Agriculture and Life Sciences, Chonnam National University, Gwangju \\ 500-757, Korea \\ ${ }^{2}$ Molecular Phytobacteriology Laboratory, Superbacteria Research Center, KRIBB, Daejeon 305-806, Korea \\ ${ }^{3}$ Biosystems and Bioengineering Program, University of Science and Technology (UST), Daejeon 305-333, Korea
}

(Received on October 31, 2013; Revised on January 21, 2014; Accepted on February 17, 2014)

Plant has possessed diverse stress signals from outside and maintained its fitness. Out of such plant responses, it is well known that mitogen-activated protein kinase (MAPK) cascade plays important role in wounding and pathogen attack in most dicot plants. However, little is understood about its role in wounding response for the economically important monocot rice plant. In this study, therefore, the involvement of MAPK was investigated to understand the wounding signaling pathway in rice. The OsMPK1 was rapidly activated by wounding within $10 \mathrm{~min}$, and OsMPK1 was also activated by challenge of rice blast fungus. Further analysis revealed that OsMKK4, the upstream kinase of OsMPK1, phosphorylated OsMPK1 by wounding in vivo. Furthermore, OsMPK1 directly interacted with a rice defense-related transcription factor OsWRKY53. To understand a functional link between MAPK and its target transcription factor, we showed that OsMPK1 activated by the constitutively active mutant OsMK$\mathrm{K} 4^{\mathrm{DD}}$ phosphorylated OsWRKY53 in vitro. Taken together, components involving in the wounding signaling pathway, OsMKK4-OsMPK1-OsWRKY53, can be important players in regulating crosstalk between abiotic stress and biotic stress.

Keywords : defense response, MAPK cascade, rice, wounding signaling pathway, WRKY transcription factor

Plant wounding response must be a rapid and coordinated process in the local region of injury, since wounded

*Corresponding author.

Phone) +82-62-530-2076, FAX) +82-62-530-0207

E-mail) kyyang@chonnam.ac.kr sites are known to be the main entry points for all kinds of plant pathogens, such as fungi, bacteria and viruses (Bostock and Stermer, 1989). Therefore, plants have evolved mechanisms which integrate the wounding and pathogen response. In fact, wounding response overlaps extensively with pathogen response, and both responses involve a number of the same plant hormones, such as jasmonic acid (JA), salicylic acid (SA) and ethylene (ET) (Cheong et al., 2002; Reymond and Farmer, 1998). Therefore, research associated with the signal transduction of plant wounding response is useful to understand the plant defense response against biotic stress. However, most of these studies have been based on dicot plants, and as such, these processes are less-well understood for monocot plants, such as rice.

The mitogen-activated protein kinase (MAPK) cascade is a major and evolutionally conserved signal pathway, which is a major component downstream of receptors/ sensors and transduces extracellular stimuli into intracellular responses in eukaryotic cells (Jonak et al., 2002). MAPK cascades are three protein kinase component system, MAPK, MAPK kinase (MAPKK) and MAPKK kinase (MAPKKK). Extensive studies have shown that salicylic acid-induced protein kinase (SIPK), and woundinduced protein kinase (WIPK) in tobacco, and their functional orthologs of Arabidopsis MPK6 and MPK3, respectively, which are induced by response to wounding, fungal and bacterial pathogens (Cheong and Kim, 2010; Rodriguez et al., 2010). It was identified that NtMEK2, a tobacco MAPK kinase, is the upstream kinase of SIPK and WIPK, and was found to induce the production of ethylene, defense-related gene expression, and HR-like cell death (Yang et al., 2001). In Arabidopsis, MKK4 and MKK5, two Arabidopsis orthologs of NtMEK2, activate downstream MAPKs MPK3/MPK6 (Ren et al., 2002). MPK3/MPK6 regulates the biosynthesis of camalexin 
by phosphorylation of the WRKY33 transcription factor, which promotes the expression of camalexin biosynthetic genes (Mao et al., 2011). In case of rice, OsMPK1 and OsMPK5 are the most characterized among all of the 17 rice MAPKs. OsMPK1 is also called as OsSIPK and OsMPK6, which is a functional orthologs of tobacco SIPK and Arabidopsis MPK6 (Rohila et al., 2007). OsMPK5 is also called as OsMAPK5, OsBIMK1 and OsMPK3, which is a functional orthologs of tobacco WIPK and Arabidopsis MPK3 (Rohila et al., 2007). The expression of $N t M E K 2^{D D}$, which encodes the constitutively active mutant of NtMEK2, in NtMEK2 ${ }^{D D}$ transgenic rice plants activates the endogenous rice 48-kDa MAPK and leads to HR-like cell death, which was preceded by the generation of $\mathrm{H}_{2} \mathrm{O}_{2}$ (Jeong et al., 2008). In addition, chitin elicitor activates two rice MAPKs (OsMPK1 and OsMPK5) and one MAPK kinase (OsMKK4), and OsMKK4-OsMPK6 (OsMPK1) cascade mediates a fungal chitin elicitor signal and regulates defense responses, including antimicrobial biosynthesis, leading to plant cell death without extracellular reactive oxygen species (ROS) production (Kishi-Kaboshi et al., 2010). These evidences suggest that both dicot and monocot plants have a similar conserved MAPK cascade to that of the tobacco NtMEK2-SIPK/WIPK pathway. However, wounding-responsive MAPK cascade and downstream substrates of MAPK are poorly understood in rice.

In this study, therefore, we demonstrate that OsMKK4 acts as the upstream kinase for OsMPK1, both in vitro and in vivo, after wounding. In addition, OsMPK1 directly interacts with OsWRKY53 and OsMPK1 activated by the constitutively active mutant OsMKK $4^{\mathrm{DD}}$ phosphorylates OsWRKY53 in vitro. Taken together, our results suggest that OsWRKY53 may function downstream of woundingresponsive OsMKK4-OsMPK1 cascade in rice.

\section{Materials and Methods}

Plant materials and treatments. Wild-type (Oryza sativa L. cv. Dongjin), NtMEK2 ${ }^{D D}$ and NtMEK2 ${ }^{K R}$, constitutively active mutant and inactive mutant of NtMEK2, respectively, transgenic rice seeds were surface-sterilized and germinated. Leaves were wounded using scissors, and Magnaporthe grisea-infected leaves were harvested at the indicated time points. The detached 8-week-old NtMEK2 $2^{D D}$ transgenic rice leaves were placed in a solution containing $100 \mu \mathrm{M}$ DEX for $48 \mathrm{~h}$ and were then collected.

Antibody production, in vitro pull down assay, immunoblot analysis and in-gel kinase activity assay. Polyclonal specific antibodies were raised in rabbits against amino acids 1 to 30 of OsMKK4, and amino acids 61 to 100 of OsMPK5. However, the antibody for OsMPK1 was finally raised against the whole protein of OsMPK1 purified from E. coli, because antibody with peptides for OsMPK1 showed non-specific binding to the different proteins. After affinity purification, the specificity and cross-reactivity of these antibodies were assessed by using immunoblot analysis. Immunoblot analysis using anti-Flag, anti-Histidine, anti-GST, anti-OsMKK4, anti-OsMPK1, and anti-OsMPK5 were carried out as described previously (Jeong et al., 2008). In-gel kinase activity assay was performed with myelin basic protein (MBP) as a substrate for kinase as described previously (Jeong et al., 2008). Briefly, $15 \mu \mathrm{g}$ of protein extracts were electrophoresed on $10 \%$ SDS-polyacrylamide gels embedded with $0.1 \mathrm{mg} / \mathrm{ml}$ MBP in separating gel as a substrate for the kinases. After electrophoresis, the SDS was removed from the gel by washing with washing buffer ( $25 \mathrm{mM}$ Tris-HCl, $\mathrm{pH}$ 7.5, 0.5 $\mathrm{mM}$ dithiothreitol, $0.1 \mathrm{mM} \mathrm{Na}_{3} \mathrm{VO}_{4}, 5 \mathrm{mM} \mathrm{NaF}, 0.5 \mathrm{mg} /$ $\mathrm{ml}$ bovine serum albumin and $0.1 \%$ Triton X-100) three times for $30 \mathrm{~min}$ each at room temperature. The proteins were then renatured in buffer $(25 \mathrm{mM}$ Tris- $\mathrm{HCl}, \mathrm{pH} 7.5$, $1 \mathrm{mM}$ dithiothreitol, $0.1 \mathrm{mM} \mathrm{Na}_{3} \mathrm{VO}_{4}$ and $5 \mathrm{mM} \mathrm{NaF}$ ) at $4^{\circ} \mathrm{C}$ overnight with three changes of buffer. The gel was then incubated in $100 \mathrm{ml}$ of reaction buffer $(25 \mathrm{mM}$ Tris$\mathrm{HCl}, \mathrm{pH}$ 7.5, 2 mM EGTA, $12 \mathrm{mM} \mathrm{MgCl}_{2}, 1 \mathrm{mM}$ dithiothreitol and $0.1 \mathrm{mM} \mathrm{Na}_{3} \mathrm{VO}_{4}$ ) at room temperature for 30 min. Next, phosphorylation was per formed for $1.5 \mathrm{~h}$ at room temperature in $30 \mathrm{ml}$ of the same reaction buffer that contained $200 \mathrm{nM}$ ATP plus $50 \mathrm{Ci}$ of $\left[\gamma_{-}{ }^{32} \mathrm{P}\right] \mathrm{ATP}(3000 \mathrm{Ci} /$ mmol). The reaction was then stopped by transferring the gel into a solution that contained $5 \%$ trichloroacetic acid (w/ $\mathrm{v})$ and $1 \%$ sodium pyrophosphate $(\mathrm{w} / \mathrm{v})$. The unincorporated radioactivity was subsequently removed by washing the gel for $6 \mathrm{~h}$ at room temperature, with five changes. The gel was then dried on $3 \mathrm{M}$ paper and subjected to autoradiography. Prestained size markers were used to calculate the size of the kinases.

In vitro pull down assay between His-tagged OsMPK1 and GST-tagged recombinant proteins, GST only, GSTOsWRKY24, GST-OsWRKY53, and GST-OsWRKY78, were performed by using glutathione-agarose $50 \%$ slurry according to the manufacturer's instructions (GE health care). Proteins precipitated with glutathione-agarose complex were immunoblotted with anti-Histidine antibody.

Reverse transcription-polymerase chain reaction (RTPCR) and quantitative real-time polymerase chain reaction (qRT-PCR). Reverse transcription PCR and qRT-PCR was performed as described previously (Jeong 
Table 1. Gene specific primer pairs used for RT-PCR and qRT-PCR

\begin{tabular}{cll}
\hline Gene & \multicolumn{1}{c}{ Forward primer $\left(5^{\prime}-3^{\prime}\right)$} & \multicolumn{1}{c}{ Backward primer (5'-3') } \\
\hline OsWRKY24 & GACCTGAGGGATTCCGATGAC & CGACGGGAAGATACTGGAGG \\
OsWRKY53 & CCGTTCATCACGTCGTTCAC & GGTCGGGGACGCCAAGATA \\
OsWRKY78 & TACAGGATGCCCAGTCAGGA & AGGCCCTGCATATTGCTGTT \\
OsWRKY45 & CGGGTAAAACGATCGAAAGA & CCCCAGCTCATAATCAGGAA \\
I 8 S $R$ R $N A$ & ATGATAACTCGACGGATCGC & CTTGGATGTGGTAGCCGTTT \\
\hline
\end{tabular}

et al., 2008; Kim et al., 2013). In brief, qRT-PCR with the QuantiTect SYBR Green RT-PCR kit (JMC R\&D) was conducted using a Rotor-Gene 2000 real-time thermal cycling system (Corbett Research). The reaction mixture (20 $\mu \mathrm{l})$ contained $1 \mu \mathrm{l}$ of $100 \mathrm{ng}$ total RNA, $0.5 \mu \mathrm{l}$ of $0.5 \mathrm{pmol}$ specific primer, as well as appropriate amounts of enzymes and fluorescent dyes, in accordance with the manufacturer's instructions. After normalization to an $18 S \mathrm{rRNA}$ control, the relative levels of gene expression were calculated. The primer pairs are listed in Table 1.

Plasmid construction and purification of recombinant proteins. The full-length cDNA clones of OsMPK1 and OsMKK4 (Accession No. AK111942 and AK120525, respectively) were kindly obtained from the Rice Genome Resource Center (http://www.rgrc.dna.affrc.go.jp) and the each cDNA was amplified by PCR with the provided clones as templates. The OsMPK5, OsWRKY24, OsWRKY53, and OsWRKY78 cDNA clones used in this study were isolated by reverse transcription-PCR and were cloned into pGEM-T easy vector.

The constitutively active and inactive mutants of OsMKK4, as well as the inactive mutant of OsMPK1, were generated by site-directed mutagenesis, and ligated in frame into the pET-28a $(+)$ vector (Novagen, USA). BL21(DE3) cells transformed with pET-28a $(+)$ constructs were induced with $0.5 \mathrm{mM}$ isopropylthio- $\beta$-thiogalactoside for $3 \mathrm{~h}$. Histagged proteins were purified using nickel columns (Qiagene, Germany) and were concentrated using Centricon-10 (Millipore, USA). The OsWRKY24, OsWRKY53, and OsWRKY78 cDNA were constructed to pGEX4T vector for expressing the recombinant proteins. GST-tagged proteins were purified using glutathione-agarose $50 \%$ slurry according to the manufacturer's instructions (GE health care).

Kinase assays. Immunocomplex (IC)-kinase assay of OsMPK1 and OsMPK5 were performed with OsMPK1specific (Anti-OsMPK1) and OsMPK5-specific (AntiOsMPK5) antibody, respectively, by using protein extracts after wounding treatment. After immunoprecipitation, the protein kinase activity was measured by adding MBP as a substrate as previously described (Yang et al., 2001). Phosphorylation activities of OsMKK4 and its active mutant OsMKK $4{ }^{\mathrm{DD}}$ were determined by using OsMPK1 and its inactive mutants OsMPK $1^{\mathrm{KR}}$ as substrates. Protein extracts from wounding treated rice plant were immunoprecipitated with purified OsMKK4-specific (anti-OsMKK4) antibody in immunoprecipitation buffer. Kinase activity of the immunocomplex was subsequently assayed with His-OsMP$\mathrm{K}^{\mathrm{KR}}$ as a substrate. In vitro phosphorylation assay for OsWRKY53 was carried out by first incubating His-OsMPK1 $(1 \mu \mathrm{g})$ with $0.1 \mu \mathrm{g}$ of His-OsMKK $4^{\mathrm{DD}}$ in the presence of 50 $\mu \mathrm{M}$ unlabeled ATP at $30^{\circ} \mathrm{C}$ for $15 \mathrm{~min}$. GST-OsWRKY53 recombinant protein (final concentration of $0.25 \mu \mathrm{g} / \mu \mathrm{l}$ ) and $\left[\gamma-{ }^{32} \mathrm{P}\right] \mathrm{ATP}(1 \mu \mathrm{Ci}$ per reaction) were then added, and the reactions were stopped by the addition of SDS loading buffer after $30 \mathrm{~min}$. The phosphorylated GST-OsWRKY53 was visualized by autoradiography after being resolved on a 15\% SDS-polyacrylamide gel (Yang et al., 2001).

Agrobacterium-mediated transient transformation of tobacco plants. Tobacco plants (Nicotiana tabacum cv Xanthi nc [NN] were grown at $25^{\circ} \mathrm{C}$ in a growth chamber programmed for a 14-h light cycle. Six to seven-weekold tobacco plants were used for transient transformation. Agrobacterium tumefaciens-mediated transient transformation experiments with $N t M E K 2^{D D}$ construct and three different OsMKK4 constructs were performed as described previously (Yang et al., 2001).

\section{Results and Discussion}

The 48-kDa MAPK activated in rice leaves by wounding is OsMPK1, which is also activated by rice blast fungus. Previously Katou and co-workers suggested that the activities of both OsMPK3 (OsMPK5) and OsMPK6 (OsMPK1) were transiently increased in rice by wounding within $10 \mathrm{~min}$ and returned to nearly the basal level 30 min after wounding by using immunocomplex (IC)-kinase assay (Katou et al., 2007). However, we found that under 
A

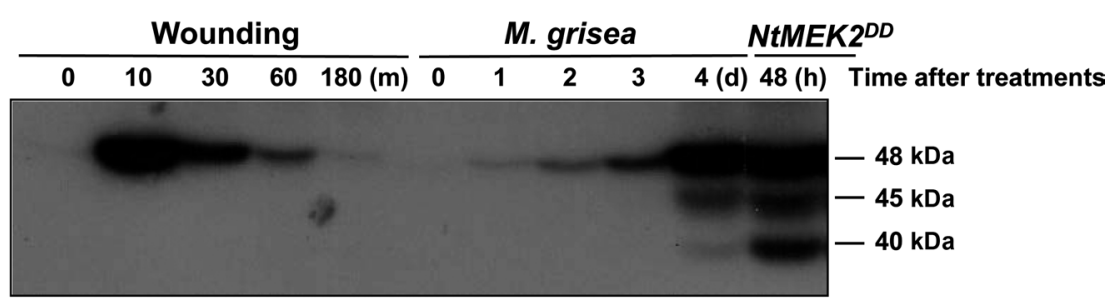

B

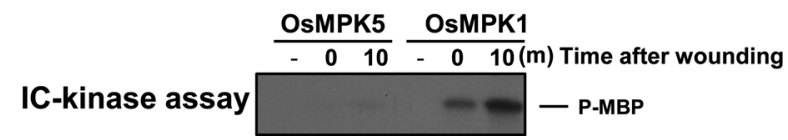

Fig. 1. Identification of wounding-responsive OsMPK1. (A) The activation of 48-kDa MAPK in rice after the treatment of mechanical wounding $(0,10,30,60$, and $180 \mathrm{~min})$ was determined by in-gel kinase assay with MBP as a substrate. Protein extracts from $M$. grisea-infected $\left(0,1,2,3\right.$, and 4 day) rice leaves and $N t M E K 2^{D D}$ transgenic rice plants after DEX treatment (48 h) were also collected and analyzed by in-gel kinase assay. (B) The 48-kDa MAPK activated by wounding corresponds to OsMPK1. To perform an immunocomplex (IC)-kinase assay, protein extracts from untreated and wounding-treated (10 min) rice leaves were immunoprecipitated with the OsMPK1-specific (anti-OsMPK1) or OsMPK5-specific (anti-OsMPK5) antibody. The kinase activity of immunocomplex was determined by an in-solution kinase assay with MBP as a substrate, and the phosphorylated MBP was visualized by autoradiography.

our experimental conditions, activation of the MAPK of about $48-\mathrm{kDa}$ was activated rapidly at $10 \mathrm{~min}$ after mechanical wounding treatment with scissors and the MAPK activity was decreased within $180 \mathrm{~min}$ to the basal level by using in-gel kinase assay with MBP as a substrate (Fig. 1A). The activation of the same $48-\mathrm{kDa}$ MAPK was also observed as the top band among three bands in rice leaves that were treated with rice blast fungus, Magnaporthe grisea, and in NtMEK2 $2^{D D}$ transgenic rice, respectively. As shown in Fig. 1A, a 48-kDa MAPK was quickly and transiently activated by wounding, but the activation of a 48-kDa, and other a 45-kDa, and a 40-kDa MAPK were observed 4 days after inoculation with rice blast fungus, and $48 \mathrm{~h}$ after dexamethasone (DEX) treatment in NtME$K 2^{D D}$ transgenic rice. To confirm whether $48-\mathrm{kDa}$ MAPK is activated by wounding, we raised the antibodies directed against peptides corresponding to the unique $\mathrm{N}$ termini of OsMPK1 and OsMPK5. However, antibody with peptides for OsMPK1 showed non-specific binding to the different proteins. Therefore, the antibody for OsMPK1 was finally raised against the whole protein of OsMPK1 purified from $E$. coli. The specificity of the anti-OsMPK1 and antiOsMPK5 were assessed by immunoblot analysis against different His-tagged recombinant OsMKK4, OsMPK1, OsMPK5, and OsMPK12 proteins. The anti-OsMPK1 only reacted with His-OsMPK1, and not with His-OsMKK4, His-OsMPK5, or His-OsMPK12. Furthermore, the antiOsMPK5 only reacted with His-OsMPK5, and not with His-OsMKK4, His-OsMPK1, or His-OsMPK12 (Supplementary Fig. 1).
To perform an IC-kinase assay, protein extracts from leaves treated with wounding for 10 min were reacted with the OsMPK1 specific antibody anti-OsMPK1. The 48-kDa MAPK activated by wounding was immunoprecipitated by the OsMPK1-specific antibody (Fig. 1B), suggesting that it is OsMPK1. An IC-kinase assay was also subjected with OsMPK5 specific antibody anti-OsMPK5 to determine whether $48-\mathrm{kDa}$ MAPK was activated by wounding to react with OsMPK5 or not. However, 48-kDa MAPK was not immunoprecipitated by the OsMPK5-specific antibody (Fig. 1B). These results suggest that the rice woundingresponsive 48-kDa MAPK is OsMPK1. However, activation of OsMPK1 by wounding was not associated with an increase in mRNA or protein levels, suggesting that OsMPK1 is posttranslationally activated by wounding response (data not shown). These results are consistent with a study which was conducted by Lee et al. (2008), in which OsSIPK (OsMPK1) mRNA is strongly induced by jasmonic acid (JA), salicylic acid (SA), and some other stresses, but not with wounding by cut. Furthermore, the mRNA levels of OsMAPK6 (OsMPK1) and OsMAPK6 (OsMPK1) protein levels remained constant and did not change in response to the sphingolipid elicitor (Lieberherr et al., 2005).

Early, Zhang and Klessig (1998) demonstrated in tobacco that various mechanical stresses, including cutting and wounding by abrasion, activates a $48-\mathrm{kDa}$ MAPK, which is SIPK, rather than WIPK, by using an immunoprecipitation complex kinase assay. Another report also suggested in soybean cells that wounding activated a 49-kDa MAPK alone when the kinase activity was tested by an in-gel ki- 
nase assay, and this MAPK was immunoprecipitated by the alfalfa MAPK, SIMK, which corresponds to tobacco SIPK and Arabidopsis AtMPK6 (Lee et al., 2001). In this study, however, since we determined different results to those of Katou and co-workers, further detailed work needs to be done to fully understand the biochemical mechanisms of OsMPK1 and OsMPK5 involved in wounding signaling in rice.

It has been demonstrated that tobacco wounding- and fungal elicitors-activated SIPK was involved in both disease resistance and response to wounding (Zhang and Klessig, 1998). In this study, OsMPK1 was also activated by wounding, rice blast fungus, and constitutively active mutant of NtMEK2, which is the upstream kinase of SIPK and WIPK. Furthermore, in rice, Cho and co-workers demonstrated that OsSIPK (OsMPK1) functions as a "central master switch" for integrating and converting numerous signals derived from external stimuli for adjusting physi-

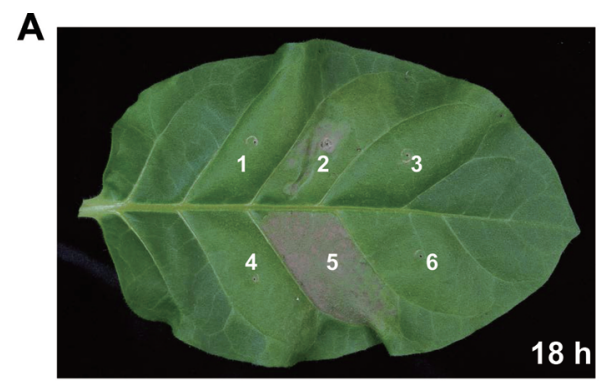

\begin{tabular}{|c|c|c|}
\hline & Constructs & $\begin{array}{c}18 \mathrm{~h} \text { after DEX } \\
\text { treatment }\end{array}$ \\
\hline 1 & OsMKK4 ${ }^{\mathrm{WT}}$ & - \\
\hline 2 & OsMKK4 $4^{\mathrm{DD}}$ & HR (small ) \\
\hline 3 & OsMKK4 $4^{\mathrm{KR}}$ & - \\
\hline 4 & pTA vector & - \\
\hline 5 & NtMEK2 $2^{\text {DD }}$ & HR (almost whole) \\
\hline \multirow[t]{2}{*}{6} & pTA vector & - \\
\hline & Constructs & $\begin{array}{c}36 \text { h after DEX } \\
\text { treatment }\end{array}$ \\
\hline 1 & OsMKK4 ${ }^{W T}$ & - \\
\hline 2 & OsMKK4 $4^{\mathrm{DD}}$ & HR (complete whole) \\
\hline 3 & OsMKK $4^{\mathrm{KR}}$ & - \\
\hline 4 & pTA vector & - \\
\hline 5 & NtMEK2 $2^{\text {DD }}$ & HR (complete whole) \\
\hline 6 & pTA vector & - \\
\hline
\end{tabular}

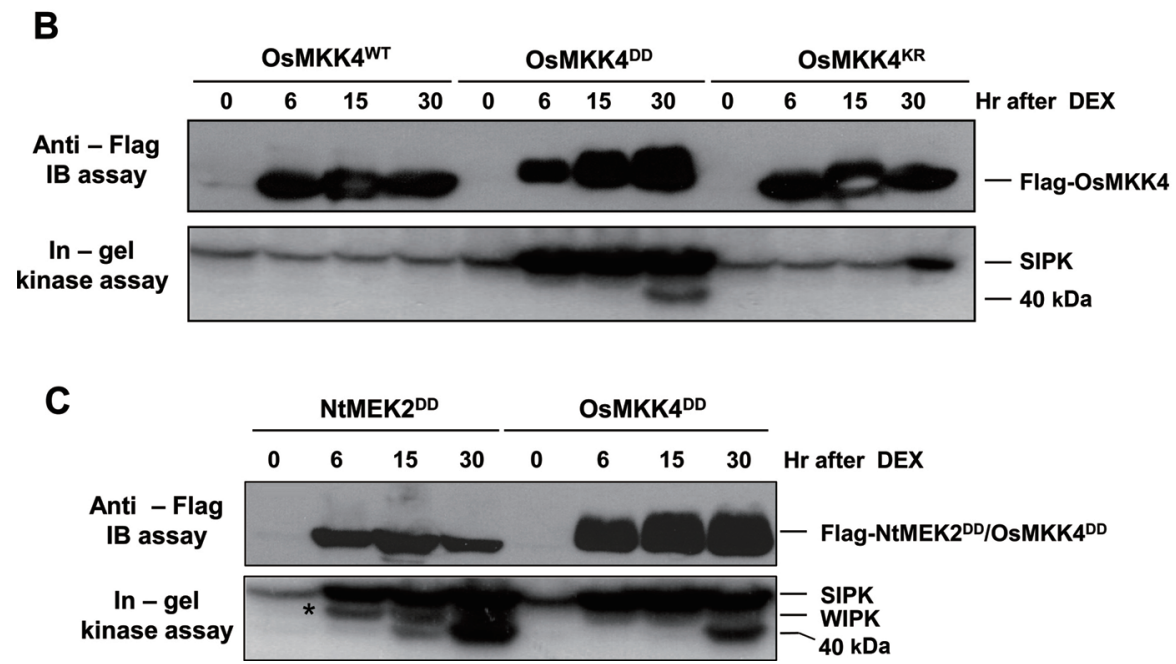

C

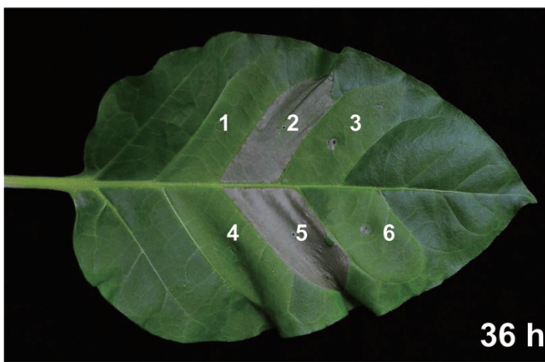

Fig. 2. Expression of OsMKK $4^{\mathrm{DD}}$ induces HR-like cell death and activates SIPK in tobacco. (A) Agrobacterium carrying the indicated constructs in pTA7002 vector was transiently transformed into different sections of tobacco leaves. Two days later, the transgene expression was induced by the application of DEX $(30 \mu \mathrm{M})$. Photographs were taken at 18 and $36 \mathrm{~h}$ after DEX treatment. In the table, 'HR' denotes the development of HR-like cell death, and '-' indicates no visible phenotype. (B and C) Induction of OsMKK4 ${ }^{\mathrm{DD}}$ expression activates SIPK in tobacco. To confirm the activation of tobacco SIPK and WIPK, NtMEK2 ${ }^{\text {DD }}$ construct was used as a positive control. Expression of transgenes was monitored by immunoblot analysis (IB) by using anti-Flag antibody. The MAPK activities after DEX treatment were determined by an in-gel kinase assay with MBP substrate. The band corresponding to WIPK is indicated with asterisks $\left({ }^{*}\right)$. All experiments were repeated two times with similar results. 
ological responses (Cho et al., 2009). Taken together, these results suggest that OsMPK1 may coordinate several distinct pathways that lead to disease resistance responses and wounding responses in rice.

OsMPK1 is the substrate of OsMKK4 in the wounding signaling pathway of rice. We demonstrated that the 48-kDa MAPK activation, which is OsMPK1, mainly occurred in $N t M E K 2^{D D}$ transgenic rice after DEX treatment (Fig. 1A). This result indicates that tobacco NtMEK2 can phosphorylate and activate rice MAPKs, especially OsMPK1. Thus, to investigate the MAPK cascade that mediates the wounding signaling pathway of rice, we identified OsMKK4 as a rice ortholog of tobacco NtMEK2 on the basis of their amino acid homology and generated the constitutively active or inactive mutants, OSMKK $4^{D D}$ or $O s M K K 4^{K R}$, respectively, under the control of a DEXinducible promoter. Within 15-18 $\mathrm{h}$ after the application of DEX, cell death started to appear in small areas infiltrated with Agrobactrium carrying $O s M K K 4^{D D}$, however, the cell death in almost whole areas infiltrated with Agrobactrium carrying $N t M E K 2^{D D}$ could be seen (Fig. $2 \mathrm{~A}$ upper). By 32-36 h, the whole area infiltrated with Agrobacterium carrying $O s M K K 4^{D D}$ collapsed, as $N t M E K 2^{D D}$ did (Fig. 2A lower). In contrast, the $O s M K K 4^{W T}$ and $O s M K K 4^{K R}$, a wildtype and an inactive mutant, respectively, did not show cell death, even though these proteins were expressed at a similar level (Fig. 2B). The HR-like cell death phenotype is correlated with high level of activation of SIPK in tobacco. However, the cell death phenotype induced by $O S M K K 4^{D D}$ was weaker and delayed compared to $N t M E K 2^{D D}$, which correlates with the non-activation of WIPK (Fig. 2C). This data is consistent with the results of a study performed by Zhang and Liu (2001) in which SIPK alone was found to be sufficient for the activation of defense responses, however, the HR-like cell death phenotype was delayed in the absence of WIPK activity. Furthermore, extensive research on interaction between SIPK and WIPK demonstrated that the co-expression of WIPK with the constitutively active mutant of NtMEK2 resulted in the high-level activation of WIPK, which leads to accelerated HR-like cell death in tobacco (Liu et al., 2003). These results indicate that OsMKK4 can phosphorylate and activate tobacco SIPK, which is the tobacco ortholog of rice OsMPK1.

Therefore, we tested whether OsMPK1 is a downstream substrate of OsMKK4. If OsMKK4 functions as the upstream kinase of OsMPK1, the OsMKK4 should be able to phosphorylate and activate OsMPK1. First, to test whether OsMKK4 is able to phosphorylate OsMPK1, inactive His-OsMPK $1^{\mathrm{KR}}$ proteins with the catalytic essential Lys
(K) residue substituted with an $\operatorname{Arg}(\mathrm{R})$ were used as a substrate. His-OsMPK $1^{\mathrm{KR}}$ proteins lack autophosphorylation activity, and as such, are ideal substrates for assaying MAPKK activity (Yang et al., 2001). As shown in Fig. $3 \mathrm{~A}$, when His-OsMKK $4^{\mathrm{WT}}$ and His-OsMKK $4^{\mathrm{DD}}$ proteins are present, His-OsMPK $1^{\mathrm{KR}}$ protein is phosphorylated, and is especially strongly phosphorylated by His-OsMKK $4^{\text {DD }}$ compared to His-OsMKK $4^{\text {WT }}$. Furthermore, phosphorylation of His-OsMPK $1^{\text {WT }}$ by His-OsMKK $4^{\text {DD }}$ enhanced their activities toward MBP as a substrate (Fig. 3B). Also, phosphorylation of His-OsMPK $1^{\mathrm{KR}}$ in vivo is correlated with MAPKK activity of Flag-NtMEK2 ${ }^{\text {DD }}$ in NtMEK2 $2^{D D}-5-1$ and $N t M E K 2^{D D}-5-4$ transgenic rice plants after $48 \mathrm{~h}$ of DEX treatment, as investigated by IC-kinase assay (Fig. 3C). However, Flag-NtMEK2 $2^{\text {KR }}$ expressed in NtMEK2 ${ }^{K R}{ }_{-6}-3$ and $N t M E K 2^{K R}{ }^{K}-6-7$ transgenic rice plants after $48 \mathrm{~h}$ of DEX treatment do not activate His-OsMPK $1^{\mathrm{KR}}$, even though the proteins were expressed (Fig. 3C). Therefore, NtMEK2 is functionally interchangeable with OsMKK4 for the activation of downstream MAPK in rice. These findings suggest that OsMKK4 might be the upstream kinase for OsMPK1 in vitro, and that NtMEK2 can phosphorylate the endogenous OsMPK1 in vivo. Since we determined that OsMKK4 is the upstream kinase of OsMPK1, we decided to investigate the interaction of OsMKK4 and OsMPK1 in wounding signaling pathway of rice, and protein extracts of leaves treated with wounding for $10 \mathrm{~min}$ were reacted with the OsMKK4 specific antibody anti-OsMKK4 and IC-kinase assay was performed with His-OsMPK ${ }^{\mathrm{KR}}$ as substrates. As shown in Fig. 3D, the recombinant protein of His-OsMPK $1^{\mathrm{KR}}$ could be phosphorylated after wounding treatment. This result suggests that OsMKK4 can phosphorylate the OsMPK1 by wounding in vivo, and that OsMKK4 is upstream kinase of OsMPK1 in the wounding signaling pathway.

The entire MAPKKK-MAPKK-MAPK cascade for the signaling of several abiotic and biotic stresses has been demonstrated from numerous studies in dicot plants, such as Arabidopsis and tobacco. However, the stress-responsive entire MAPK cascade of monocot plants, especially model crop rice, is less well-discussed. Recently, the OsMKK4OsMPK6 (OsMPK1) cascade that plays a crucial role in reprogramming plant metabolism during chitin elicitortriggered defense response of rice (Kishi-Kaboshi et al., 2010). Xie and co-workers demonstrated the OsMKK6OsMPK3 (OsMPK5) cascade constitutes a moderately low-temperature signaling pathway and regulates cold stress tolerance in rice (Xie et al., 2012). Recently, a highquality mapped MAPK interaction network in rice has been described using the yeast two-hybrid system and co- 

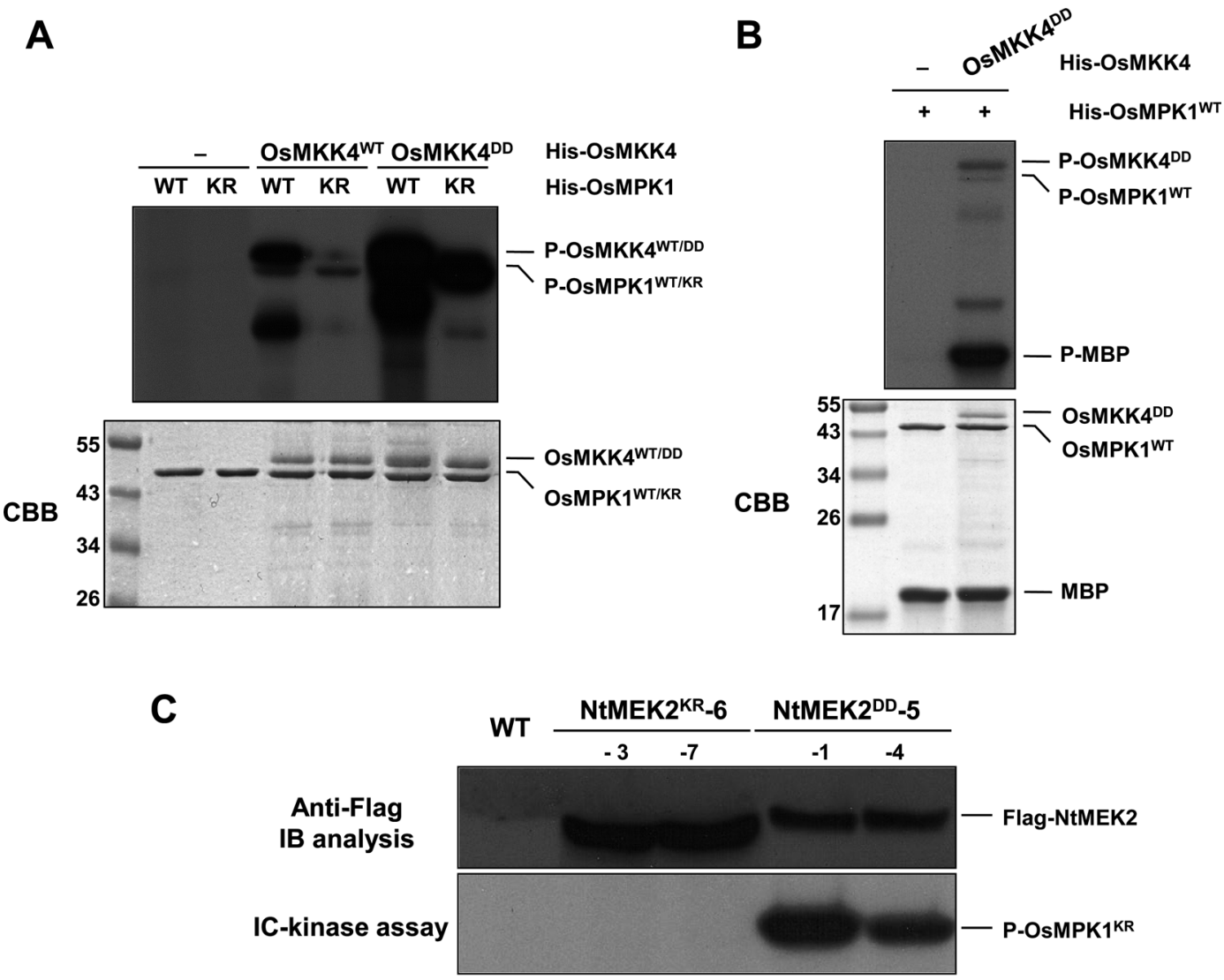

D

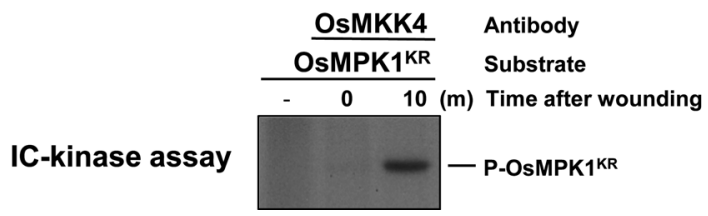

Fig. 3. OsMKK4 phosphorylates and activates OsMPK1 in vitro and in vivo after wounding. (A) OsMKK4 ${ }^{\mathrm{DD}}$, the constitutively active mutant of OsMKK4, phosphorylates OsMPK1. Phosphorylation activity of His-OsMKK4 ${ }^{\mathrm{WT}}$ and His-OsMKK4 ${ }^{\mathrm{DD}}(0.1 \mu \mathrm{g})$ were tested using inactive mutant (His-OsMPK1 ${ }^{\mathrm{KR}}, 1 \mu \mathrm{g}$ ) as a substrate. Reactions in the absence (-) of OsMKK4 were used as a control. (B) The OsMPK1 activated by OsMKK $4^{\mathrm{DD}}$ phosphorylates MBP substrate. His-OsMPK $1^{\mathrm{WT}}(1 \mu \mathrm{g})$ was incubated in the absence $(-)$ or with 0.1 $\mu \mathrm{g}$ of His-OsMKK $4^{\mathrm{DD}}$, in kinase reaction buffer including $50 \mu \mathrm{M}$ unlabeled ATP. Phosphorylated MBP (P-MBP) that indicates the activity of MAPK could be visible by autoradiography after SDS-PAGE. CBB staining of His-OsMKK4 ${ }^{\mathrm{DD}}$, His-OsMPK1 and MBP was shown in the lower panel. (C) Expression of $N t M E K 2^{D D}$ phosphorylates OsMPK1 following DEX treatment in $N t M E K 2^{D D}$ transgenic rice plants. The indicated $N t M E K 2^{K R}$ and $N t M E K 2^{D D}$ transgenic rice plants were collected $48 \mathrm{~h}$ after DEX treatment and transgene expression was analyzed by immunoblot analysis (IB) using anti-Flag antibody (Top). The kinase activities of Flag-tagged NtMEK2 ${ }^{\mathrm{KR}}$ and NtMEK2 ${ }^{\mathrm{DD}}$ were analyzed by an immunocomplex (IC)-kinase assay with His-OsMPK1 ${ }^{\mathrm{KR}}$ as a substrate. (D) OsMKK4 phosphorylates the OsMPK1 by wounding in vivo. Protein extracts treated with wounding were immunoprecipitated with OsMKK4-specific antibody anti-OsMKK4. Kinase activity of the immunocomplex was subsequently assayed with His-OsMPK1 ${ }^{\mathrm{KR}}$ as a substrate.

immunoprecipitation, pull-down, bimolecular fluorescence complementation, subcellular localization, and kinase assay experiments (Singh et al., 2012). However, only the two MAPKK-MAPK pairs, OsMKK4-OsMPK6 (OsMPK1) and OsMKK6-OsMPK3 (OsMPK5), have been functionally analyzed in rice so far (Singh and Jwa, 2013). In this study, we determined that OsMKK4 is the upstream kinase of OsMPK1 in wounding signaling pathway in rice. To our knowledge, this is the first detailed biochemical study to demonstrate the identification of the OsMKK4OsMPK1 cascade involving the wounding signaling pathway in rice. 
Phosphorylation of wounding-responsive OsWRKY53 by OsMPK1. Recently, WRKY8 was identified as a substrate of SIPK, WIPK and NTF4 in tobacco and WRKY33, the closest Arabidopsis WRKY to tobacco WRKY8, was also identified as a substrate of MPK3/MPK6 in Arabidopsis (Ishihama et al., 2011; Mao et al., 2011). To understand the downstream substrates that are regulated by the wounding-responsive OsMKK4-OsMPK1 cascade, we first analyzed the expression of WRKY genes in the NtME$K 2^{D D}$ transgenic rice plant after treatment with DEX. We selected OsWRKY24, OsWRKY53 and OsWRKY78, the closest rice WRKY to Arabidopsis WRKY33 and tobacco
WRKY8, through amino acid alignment using SP cluster and D domain. Since it was reported that OsMPK1 phosphorylated OsWRKY45 protein in vitro, we also selected OsWRKY45 as a positive control (Ueno et al., 2013). As shown in Fig. 4A, the OsWRKY24, OsWRKY53, and Os$W R K Y 45$, were highly induced in the NtMEK2 ${ }^{D D}$ transgenic rice plant within $12 \mathrm{~h}$ of treatment with DEX, whereas OsWRKY78 wasn't induced.

The interaction between OsMPK1 and OsWRKYs was confirmed by in vitro pull-down assays. OsWRKYs were glutathione S-transferase (GST)-tagged and purified in E. coli. The recombinant GST alone, $86-\mathrm{kDa}$ GST-

\section{A}

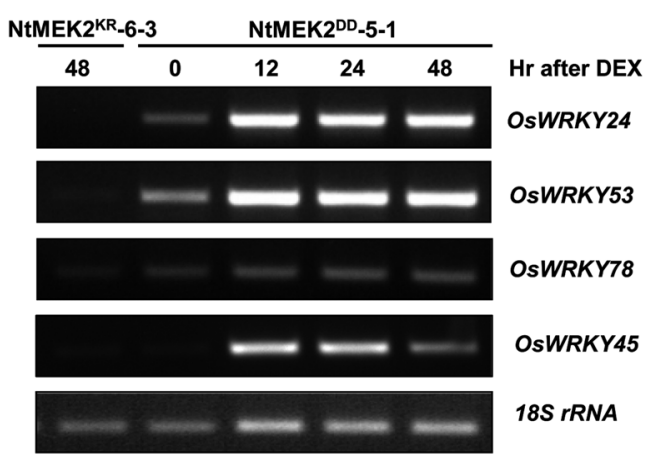

B

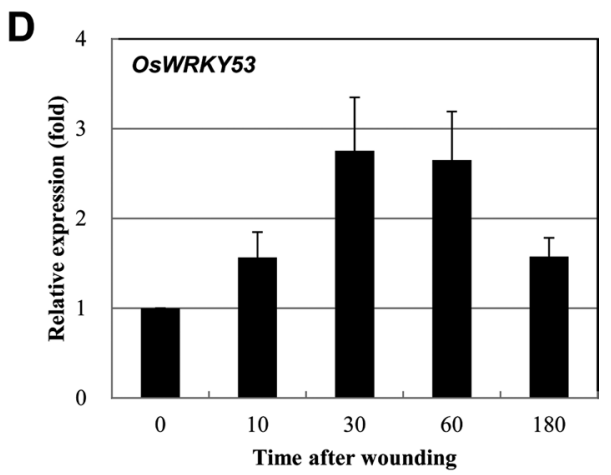

C
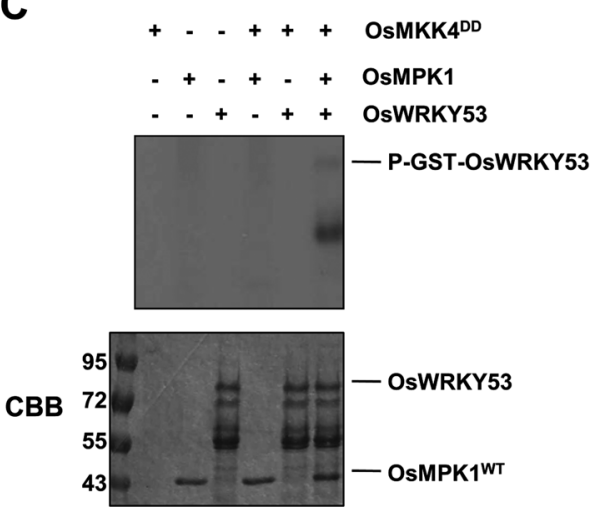

Fig. 4. OsMPK1 directly interacts with OsWRKY53 and the OsMPK1 activated by OsMKK4 ${ }^{\mathrm{DD}}$ phosphorylates OsWRKY53 in vitro. (A) Induction of $\mathrm{NtMEK}^{\mathrm{DD}}$ expression leads to the activation of OsWRKY24, OsWRKY53, and OsWRKY45. Total RNA was isolated from the samples from $N t M E K 2^{K R}{ }_{-6}-3$ and $N t M E K 2^{D D}{ }_{-}-1$ transgenic rice plants collected at the indicated times following DEX treatment, and RT-PCR was then performed. The $18 S r R N A$ was used as a loading control. (B) OsWRKY53 directly interacts with OsMPK1. In vitro pull down assay between His-tagged OsMPK1 and GST-tagged recombinant proteins, GST only, GST-OsWRKY24, GSTOsWRKY53, and GST-OsWRKY78, were performed by using glutathione-agarose. Proteins precipitated with glutathione-agarose complex were immunoblotted with anti-Histidine antibody. His-OsMPK $1^{\mathrm{WT}}$ (shown in input lane) was used as a positive control. (C) The OsMPK1 activated by OsMKK4 ${ }^{\mathrm{DD}}$ phosphorylates OsWRKY53. His-OsMPK1 ${ }^{\mathrm{WT}}(1 \mu \mathrm{g})$ was incubated in the absence $(-)$ or with $0.1 \mu \mathrm{g}$ of His-OsMKK $4^{\mathrm{DD}}$ in the kinase reaction buffer including $50 \mu \mathrm{M}$ unlabeled ATP and then the GST-OsWRKY53 recombinant protein was added. Phosphorylated GST-OsWRKY53 (P-GST-OsWRKY53) that indicates the activity of MAPK could be visible by autoradiography after SDS-PAGE. CBB staining of GST-OsWRKY53 and His-OsMPK1 was shown in the lower panel. (D) The expression of OSWRKY53 was induced after wounding $(0,10,30,60$, and $180 \mathrm{~min})$ treatment. Transcripts levels were determined by qRT-PCR. Levels of $18 S r R N A$ transcripts were used to normalize the different samples. Data shown are means \pm SE of samples conducted in triplicate. 
OsWRKY24, 77-kDa GST-OsWRKY53, or 93-kDa GST-OsWRKY78 proteins were confirmed with antiGST antibody by immunoblot analysis, even though lots of recombinant degraded forms were present (Supplementary Fig. 2). After His-OsMPK1 was incubated with glutathione-agarose-bound GST, GST-OsWRKY24, GSTOsWRKY53, or GST-OsWRKY78, respectively, and the pull-down products were detected by immunoblot analysis using anti-Histidine antibody. His-OsMPK1 was pulled down with only GST-OsWRKY53, but not GST, GST-OsWRKY24, or GST-OsWRKY78, suggesting that OsMPK1 could interact with OsWRKY53 (Fig. 4B).

To determine the phosphorylation of OsWRKY53 by OsMPK1, we applied in vitro phosphorylation assay. As shown in Fig. 4C, OsMPK1 activated by the constitutively active mutant OsMKK4 ${ }^{\mathrm{DD}}$ phosphorylated OsWRKY53. These data suggest that OsMPK1 directly phosphorylates OsWRKY53 protein in vitro. Moreover, the transcript levels of OsWRKY53 were induced dramatically by wounding (Fig. 4D). Taken together, these results suggest that OsWRKY53 may function downstream of OsMKK4OsMPK1 cascade in the wounding signaling pathway in rice.

Wounding activates large-scale changes in the expression of transcription factors, including the WRKY family in plant (Cheong et al., 2002). However, a few studies described the interaction between MAPK and WRKY transcription factor were represented in rice. Koo and coworkers suggested that OsBWMK1 (OsMPK12) leads to OsWRKY33 phosphorylation, which enhances the DNAbinding activity of OsWRKY33 and the transcription of a PR1 promoter (Koo et al., 2009). In case of OsWRKY30, phosphorylation of OsWRKY30 by MAPKs plays a crucial role in allowing OsWRKY30 to performing its biological function, which is related to drought tolerance (Shen et al., 2012). Recently, two MAPKs, OsMPK4 and OsMPK6 (OsMPK1), phosphorylated OsWRKY45 protein in vitro, and the activity of OsMPK6 (OsMPK1) was rapidly upregulated by SA treatment in rice cells (Ueno et al., 2013). However, it has not been attempted to understand the downstream substrate of MAPK cascade involving in the wounding signaling pathway in rice so far. In this study, we demonstrated that the wounding-responsive OsMKK4OsMPK1 cascade phosphorylates OsWRKY53 in vitro. Previously, Chujo et al. (2007) suggested that OsWRKY53 is induced by a fungal cerebroside elicitor and the blast fungus Magnaporthe grisea. Also several defense-related genes including PBZ1, PR-5, chitinase, and peroxidase are upregulated in rice cells overexpressing OsWRKY53, and overexpression of OsWRKY53 in rice plants results in enhanced resistance to M. grisea. As shown in Fig. 1, OsMPK1 was activated by wounding and $M$. grisea as well. Therefore, this wounding-responsive OsMKK4OsMPK1-OsWRKY53 cascade might be also involved in defense response to rice blast. The OsMKK4-OsMPK1OsWRKY53 cascade thus maybe an important player in regulating crosstalk between abiotic stress, such as wounding, and biotic stress, such as fungal pathogen. Identification of components involving in wounding signaling pathway, such as OsMKK4, OsMPK1, and OsWRKY53 transcription factor, will contribute to our understanding of how rice plants have evolved to cope with environmental stresses.

\section{Supplementary data}

Supplementary data associated with this article can be found in the online version of Plant Pathol. J.

\section{Acknowledgments}

This study was financially supported partly by Basic Science Research Program through the National Research Foundation of Korea [2009-0076547] funded by the Korean Government, and partly by the Institute of Planning and Evaluation for Technology in Food, Agriculture, Forestry and Fisheries, Ministry for Food, Agriculture, Forestry and Fisheries, Republic of Korea.

\section{References}

Bostock, R. M. and Stermer, B. A. 1989. Perspectives on wound healing in resistance to pathogens. Annu. Rev. Phytopathol. 27:343-371.

Cheong, Y. H., Chang, H.-S., Gupta, R., Wang, X., Zhu, T. and Luan, S. 2002. Transcriptional profiling reveals novel interactions between wounding, pathogen, abiotic stress, and hormonal responses in Arabidopsis. Plant Physiol. 129:661-677.

Cheong, Y. H. and Kim, M. C. 2010. Functions of MAPK cascade pathways in plant defense signaling. Plant Pathol. J. 26:101-109.

Cho, K., Agrawal, G. K., Jwa, N-S., Kubo, A. and Rakwal, R. 2009. Rice OsSIPK and its orthologs: A "Central Master Switch" for stress responses. Biochem. Biophys. Res. Commun. 379:649-653.

Chujo, T., Takai, R., Akimoto-Tomiyama, C., Ando, S., Minami, E., Nagamura, Y., Kaku, H., Shibuya, N., Yasuda, M. and Nakashita, H. 2007. Involvement of the elicitor-induced gene OsWRKY53 in the expression of defense-related genes in rice. Biochim. Biophys. Acta. 1769:497-505.

Ishihama, N., Yamada, R., Yoshioka, M., Katou, S. and Yoshioka, H. 2011. Phosphorylation of the Nicotiana benthamiana 
WRKY8 transcription factor by MAPK functions in the defense response. Plant Cell 23:1153-1170.

Jeong, J.-A., Yoo, S.-J., Yang, D.-H., Shin, S.-H., Lee, M.-C., Cho, B.-H. and Yang, K.-Y. 2008. Transgenic rice plants expressing an active tobacco mitogen-activated protein kinase kinase induce multiple defense responses. Plant Pathol. J. 24:375-383.

Jonak, C., Ökrész, L., Bögre, L. and Hirt, H. 2002. Complexity, cross talk and integration of plant MAP kinase signalling. Curr. Opin. Plant Biol. 5:415-424.

Katou, S., Kuroda, K., Seo, S., Yanagawa, Y., Tsuge, T., Yamazaki, M., Miyao, A., Hirochika, H. and Ohashi, Y. 2007. A calmodulin-binding mitogen-activated protein kinase phosphatase is induced by wounding and regulates the activities of stress-related mitogen-activated protein kinases in rice. Plant Cell Physiol. 48:332-344.

Kim, S.-H., Kim, S.-H., Yoo, S.-J., Min, K.-H., Nam, S.-H., Cho, B. H. and Yang, K.-Y. 2013. Putrescine regulating by stressresponsive MAPK cascade contributes to bacterial pathogen defense in Arabidopsis. Biochem. Biophys. Res. Commun. 437:502-508.

Kishi-Kaboshi, M., Okada, K., Kurimoto, L., Murakami, S., Umezawa, T., Shibuya, N., Yamane, H., Miyao, A., Takatsuji, H., Takahashi, A. and Hirochika, H. 2010. A rice fungal MAMP-responsive MAPK cascade regulates metabolic flow to antimicrobial metabolite synthesis. Plant J. 63:599-612.

Koo, S. C., Moon, B. C., Kim, J. K., Kim, C. Y., Sung, S. J., Kim, M. C., Cho, M. J. and Cheong, Y. H. 2009. OsBWMK1 mediates SA-dependent defense responses by activating the transcription factor OsWRKY33. Biochem. Biophys. Res. Commun. 387:365-370.

Lee, M.-O., Cho, K., Kim, S.-H., Jeong, S.-H., Kim, J.-A., Jung, Y.-H., Shim, J., Shibato, J., Rakwal, R., Tamogami, S., Kubo, A., Agrawal, G. K. and Jwa, N.-S. 2008. Novel rice OsSIPK is a multiple stress responsive MAPK family member showing rhythmic expression at mRNA level. Planta 227:981990.

Lee, S., Hirt, H. and Lee, Y. 2001. Phosphatidic acid activates a wound-activated MAPK in Glycine max. Plant J. 26:479486.

Lieberherr, D., Thao, N. P., Nakashima, A., Umemura, K., Kawasaki, T. and Shimamoto, K. 2005. A sphingolipid elicitorinducible mitogen-activated protein kinase is regulated by the small GTPase OsRac1 and heterotrimeric G-protein in rice. Plant Physiol. 138:1644-1652.

Liu, Y., Jin, H., Yang, K.-Y., Kim, C. Y., Baker, B. and Zhang, S. 2003. Interaction between two mitogen-activated protein ki- nases during tobacco defense signaling. Plant J. 34:149-160.

Mao, G., Meng, X., Liu, Y., Zheng, Z., Chen, Z. and Zhang, S. 2011. Phosphorylation of a WRKY transcription factor by two pathogen-responsive MAPKs drives phytoalexin biosynthesis in Arabidopsis. Plant Cell 23:1639-1653.

Ren, D., Yang, H. and Zhang, S. 2002. Cell death mediated by MAPK is associated with hydrogen peroxide production in Arabidopsis. J. Biol. Chem. 277:559-565.

Reymond, P. and Farmer, E. E. 1998. Jasmonate and salicylate as global signals for defense gene expression. Curr. Opin. Plant Biol. 1:404-411.

Rodriguez, M. C. S., Petersen, M. and Mundy J. 2010. Mitogenactivated protein kinase signaling in plants. Annu. Rev. Plant Biol. 61:621-649.

Rohila, J. S. and Yang, Y. 2007. Rice mitogen-activated protein kinase gene family and its role in biotic and abiotic stress response. J. Integr. Plant Biol. 49:751-759.

Shen, H., Liu, C., Zhang, Y., Meng, X., Zhou, X., Chu, C. and Wang, X. 2012. OsWRKY30 is activated by MAP kinases to confer drought tolerance in rice. Plant Mol. Biol. 80:241253.

Singh, R. and Jwa, N.-S. 2013. The rice MAPKK-MAPK interactome: the biological significance of MAPK components in hormone signal transduction. Plant Cell Rep. 32:923-331.

Singh, R., Lee, M.-O., Lee, J.-E., Choi, J., Park, J. H., Kim, E. H., Yoo, R. H., Cho, J.-I., Jeon, J.-S., Rakwal, R., Agrawal, G. K., Moon, J. S. and Jwa, N.-S. 2012. Rice mitogen-activated protein kinase interactome analysis using the yeast two-hybrid system. Plant Physiol. 160:477-487.

Ueno, Y., Yoshida, R., Kishi-Kaboshi, M., Matsushita, A., Jiang, C.-J., Goto, S., Takahashi, A., Hirochika, H. and Takatsuji, H. 2013. MAP kinases phosphorylate rice WRKY45. Plant Signal. Behav. 8:e24510.

Xie, G., Kato, H. and Imai, R. 2012. Biochemical identification of the OsMKK6-OsMPK3 signalling pathway for chilling stress tolerance in rice. Biochem. J. 443:95-102.

Yang, K.-Y., Liu, Y. and Zhang, S. 2001. Activation of a mitogenactivated protein kinase pathway is involved in disease resistance in tobacco. Proc. Natl. Acad. Sci. USA 98:741-746.

Zhang, S. and Klessig, D. F. 1998. The tobacco wounding-activated mitogen-activated protein kinase is encoded by SIPK. Proc. Natl. Acad. Sci. USA 95:7225-7230.

Zhang, S. and Liu, Y. 2001. Activation of salicylic acid-induced protein kinase, a mitogen-activated protein kinase, induces multiple defense responses in tobacco. Plant Cell 13:18771889. 